\title{
Evolution of floral symmetry
}

\author{
ENRICO S. COEN, JAGQUELINE M. NUGENT, DA LUO, \\ DESMOND BRADLEY, PILAR GUBAS, MARK GHADWICK, \\ LUGY COPSEY AND ROSEMARY CARPENTER
}

John Innes Centre, Colney, Norwich NR4 7UH, U.K.

\section{SUMMARY}

Flowers can be classified into two basic types according to their symmetry: regular flowers have more than one plane of symmetry and irregular flowers have only a single plane of symmetry. The irregular condition is thought to have evolved many times independently from the regular one: most commonly through the appearance of asymmetry along the dorso-ventral axis of the flower. In most cases, the irregular condition is associated with a particular type of inflorescence architecture. To understand the molecular mechanism and evolutionary origin of irregular flowers, we have been investigating genes controlling asymmetry in Antirrhinum. Several mutations have been described in Antirrhinum, a species with irregular flowers, that reduce or eliminate asymmetry along the dorso-ventral axis. We describe the nature of these mutations and how they may be used to analyse the molecular mechanisms underlying floral evolution.

\section{GENES GONTROLLING FLORAL SYMMETRY}

Flowers are classified as being either irregular, having only one plane of mirror symmetry or regular, having more than one plane of symmetry. The most intensive genetic analysis of floral symmetry has been carried out in Antirrhinum, which has irregular flowers that are markedly asymmetrical along their dorso-ventral axis. Wild type Antirrhinum flower have five petals that are united for part of their length to form a tube ending with five lobes. The petal lobes are of three types: two large dorsal (upper) petals; two side petals; and a ventral (lowest) petal. The flower is also irregular with respect to the stamens. Five stamens are initiated, alternate with the petals and are also of three types: the dorsal stamen is aborted and the two side stamens are shorter than the two ventral stamens. Mutations in cycloidea $(c y c)$ give regular flowers with fivefold symmetry in certain genetic backgrounds (see figure $1 a$ ). All five petals and stamens resemble the ventral petal and stamens of wild type. It has been proposed that the irregular phenotype of wild-type flowers is dependent on $c y c$ activity establishing an axis of dorso-ventral asymmetry. The activity of $c y c$ is predicted to be greatest in the dorsal regions of the flower meristem and to decline towards the more ventral regions. This would account for the ventralized phenotype of $c y c$ mutants (Carpenter \& Coen 1990). In addition to cyc, several other genes affect flower symmetry in $A n$ tirrhinum. The dichotoma mutant affects the dorsal petals and renders them more symmetrical, radialis mutants result in ventralization of the dorsal and side petals and divaricata mutants have ventral petals which resemble side petals (Stubbe 1966).

\section{EVOLUTION OF FLOWER SYMMETRY}

Primitive flowering plants are thought to have had regular flowers, and irregularity is considered to be a derived condition. The analysis of genes like $c y c$ allows us to address several important questions about the genetic basis of this evolutionary change: What role might $c y c$ have played in the ancestral species with regular flowers and how was $c y c$ subsequently recruited to establish dorsiventral asymmetry? How many times has irregularity evolved and has it always involved $c y c$ ? Before considering these questions it is first important to consider the current view of how irregularity has evolved, largely based on comparative morphology.

Irregularity is thought to have evolved independently many times, perhaps arising on as many as 25 separate occasions (Stebbins 1974). The alternative to this multiple-gain hypothesis is that irregularity arose only a limited number of times and was subsequently lost several times in independent lineages. According to this view, irregularity may be much more ancient than is commonly believed. Three types of argument can be used to evaluate these two hypotheses.

1. The multiple-gain hypothesis is claimed to be the most parsimonious way to explain the broad phylogenetic distribution of regularity as compared to the more sporadic occurrence of irregularity. However, this is partly a circular argument because the phylogenies it is based upon have been constructed using morphological data that includes floral symmetry and correlated characters. An objective evaluation can only be made if the phylogenies are independent of the morphological character being assesed. Even with a more objective phylogeny, arguments based on par- 

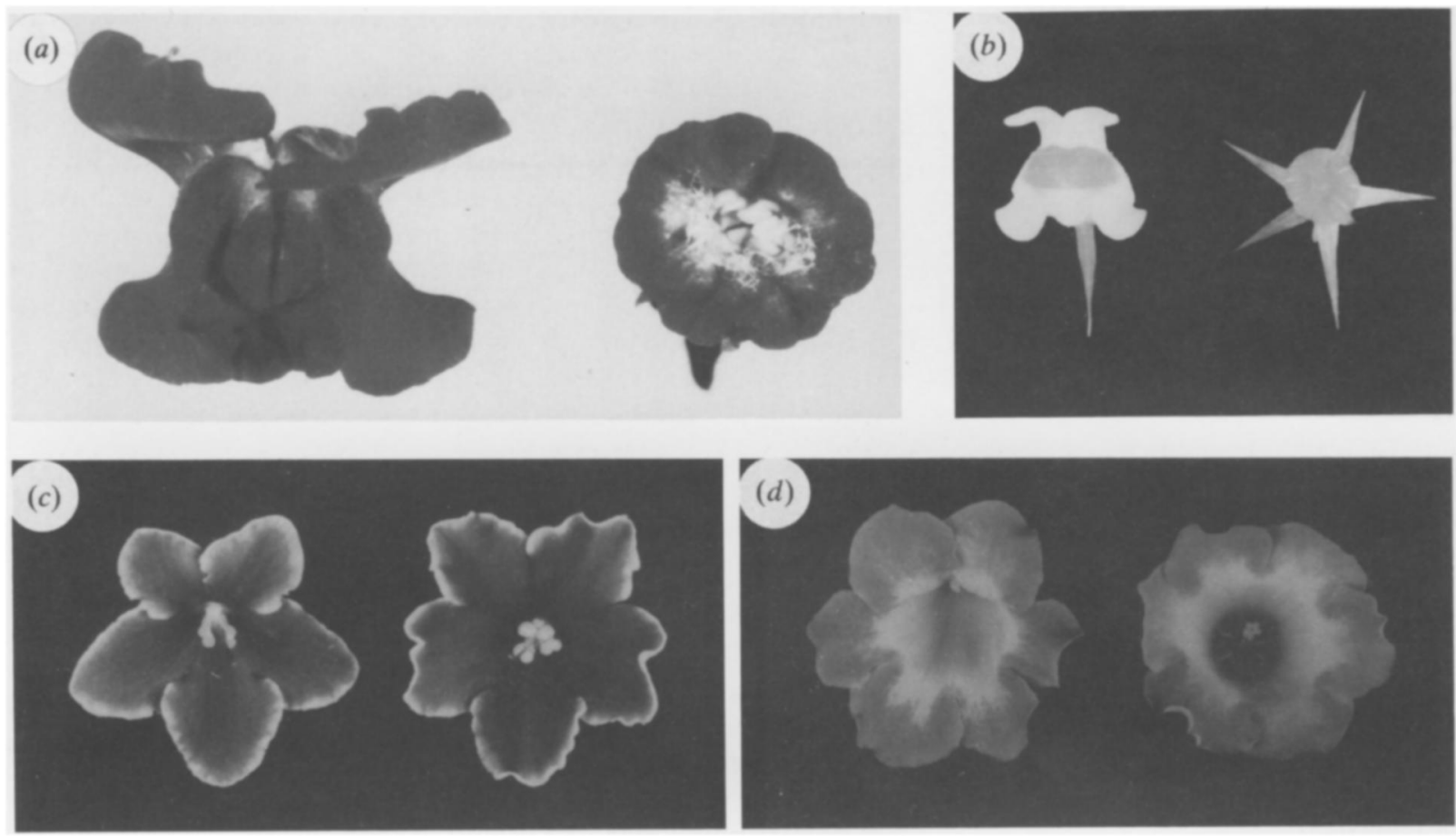

Figure 1. Wild type and cyc mutant of: (a) Antirrhinum; and comparable peloric mutants from (b) Linaria vulgaris; (c) Saintpaulia; and (d) Sinningia speciosa. For all species the wild type is on the left and the mutant on the right.

simony are problematical because they depend on knowing the relative probabilities of gaining or losing irregularity.

2. Another argument used in favour of the multiplegain hypothesis is that irregularity is a more specialized adaptation for pollination and is therefore more likely to be derived. However, although irregularity may have originally evolved from the regular state, it need not always be the derived condition. Evolution is not a unidirectional process and specialized characters can be lost as well as gained.

3. Irregular flowers occur in many different guises. For example, the irregular flowers of mint, pea and Antirrhinum are all structurally very different. This has been taken as evidence in support of the multiple-gain hypothesis as it seems to suggest that many independent mechanisms for generating irregularity have evolved. However, it is possible that some of the different types of irregular flowers share the same underlying mechanism for generating asymmetry. The differences may simply reflect the imposition of irregularity on different frameworks of floral development.

It is not possible to resolve these issues purely on the basis of taxonomic information. Isolating genes like $c y c$ that are involved in controlling floral symmetry allows a more direct approach to addressing these problems. The molecular basis of both the irregular and the regular condition could be addressed by comparing the activity of $c y c$-like genes in a range of species using a combination of genetic, molecular and transgenic techniques.

Mutants that give regular instead of irregular flowers, termed peloric mutants, have been described in several species in addition to Antirrhinum (see figure 1). The first peloric mutant was described by Linnaeus in Linaria vulgaris (toadflax), a close relative of
Antirrhinum (Linnaeus 1749). As with cyc, this mutant confers a ventralized phenotype, all petals resemble the lowest petal of wild type, which is easily distinguished in Linaria by the presence of a spur (see figure $1 b$ ). Peloric mutants with ventralized phenotypes have also been described for Saintpaulia (African Violets) and Sinningia (gloxinias), both members of the family Gesneriaceae (see figure $1 c, d$ ). Although genetic analysis of peloric mutants has not yet been carried out in species other than Antirrhinum, it is tempting to speculate that they reflect alterations in cyc-like genes. This can be tested by analysing the structure and expression of $c y c$ homologues in these mutants.

All of these examples of peloric mutants are in species that fall within a monophyletic group (the Lamiales s.l.; Olmstead et al. 1993) that includes only species with irregular flowers. It is likely that the common ancestor of this group had irregular flowers that depended on $c y c$-like gene activity. However, it is unclear whether irregularity in species that lie outside the Lamiales is also cyc-dependent. According to the multiple-gain hypothesis, the mechanism for establishing irregularity might be expected to be different outside this group. The alternative hypothesis, that irregularity is more ancient, predicts that irregularity in some species outside the Lamiales should also be cycdependent. One way to distinguish between these possibilities is to determine the role of $c y c$ homologues in species with irregular flowers such as Schizanthus (butterfly flower), Echium or even more distantly related species. If cyc-like genes are involved in controlling irregularity in these species, it then raises the question of whether $c y c$ was recruited once in a common ancestor of all these species or whether it was recruited independently several times. This might be resolved by studying the role, if any, of $c y c$-like genes in 
species with regular flowers and how this compares to its role in irregular species.

\section{GOEVOLUTION OF INFLORESGENGE ARGHITEGTURE AND FLORAL SYMMETRY}

There is a strong correlation between floral asymmetry and inflorescence architecture: irregular flowers are commonly associated with indeterminate racemose inflorescences that lack terminal flowers (Stebbins 1974). This correlation may be a consequence of either selective or developmental constraints. For example, if the adaptive value of the irregular condition depends on the flowers being presented to pollinators on a racemose inflorescence, this would mean that selection was involved. Alternatively, if the genetic mechanisms for generating asymmetry are dependent on the racemose condition, a developmental constraint would be involved.

The centroradialis (cen) mutant of Antirrhinum provides strong evidence for developmental constraints. Plants carrying the cen mutation produce a determinate inflorescence in which the main axis of growth is terminated by a single flower; unlike wild-type plants which lack a terminal flower and grow indeterminately. It is thought that in wild type, the cen gene prevents expression of genes needed for flower development in the inflorescence apex (Coen et al. 1990). A comparable mutant, terminal flower, has been described in Arabidopsis (Shannon \& Meeks-Wagner 1991; Alvarez et al. 1992). The cen mutant also affects the symmetry of flowers: axillary flowers are irregular as wild type but the terminal flower is regular. This appears to be a general property of cen-like mutants in a wide range of species with irregular flowers, such as Digitalis (Scrophulariaceae), Salvia grandiflora and Galeobdolon luteum (Labiatae), Delphinium elatum and Aconitum variegatum (Ranunculaceae); in all of these cases the terminal flower produced in the mutants is regular (Peyritsch 1870, 1872). The terminal flower of cen resembles the axillary flowers of cyc mutants, suggesting that $c y c$ is not active in the apical meristem. The production of $c y c$-dependent asymmetry seems to require that floral meristems are in axillary positions, and having a particular cellular environment. Axillary meristems are in an asymmetric environment, with the inflorescence apex above them and a subtending leaf (bract) below. This polarized environment could provide necessary cues for establishing the cyc system. By contrast, a terminal flower meristem is in a symmetrical environment and may lack the cues required to activate $c y c$ in an asymmetrical manner. Accordingly, species producing only terminal flowers might be unable to make them irregular. However, there are apparent exceptions to this view because some species, such as Schizanthus, have irregular flowers occupying terminal positions. Molecular and phenotypic analysis of these species may further our understanding of the relation between flower position and symmetry.

Other aspects of the coevolution of inflorescence type and floral symmetry appear to reflect selective constraints. Most members of the Cruciferae have regular flowers borne on extended racemes. However, in some members of this family, such as Iberis, the inflorescence axis is shorter and produces a broad dome of flowers (corymb). The overall effect of the corymb is to simulate a single large regular flower. Within the corymb flowers are irregular, the two ventral petals of each flower being much larger than the dorsal ones. This is presumably an adaptation that prevents the petals from overlapping and protruding into the centre of the inflorescence. Thus selection may have played a role in the coevolution of the compressed inflorescence axis and the irregularity of the flowers.

The coevolution of inflorescences and flowers is further illustrated by the mixed inflorescences of some of the Compositae. The inflorescence axis within this group is highly compressed to form a dense cluster of flowers (florets), termed a capitulum. In some species, such as daisy or chrysanthemum, it consists of two types of florets: ray florets that are highly irregular and occupy the periphery; and disc florets that are regular and occupy the central dome of the capitulum. This is reminiscent of the cen phenotype of Antirrhinum, where the central terminal flower is regular and the axillary flowers are irregular. In both cases, the activation of genes controlling irregularity seems to be restricted to a peripheral zone around the inflorescence apex. In the case of a daisy, this zone gives rise only to the outermost florets whereas in Antirrhinum it produces all of the axillary flowers. Nevertheless, the production of inflorescences with a mixture of regular and irregular flowers is not found as a wild-type condition in plants with extended racemes like Antirrhinum, but is common in species with a capitulum. This may reflect a selective advantage of mixed inflorescences (such as mimicking a large flower) when the flowers are tightly clustered within a capitulum.

The similarity between cen and daisy-like inflorescences raises the question of whether cyc-like genes are involved in the control of irregularity within the Compositae, a family that is quite distantly related to Antirrhinum. It is possible to test this by looking for, and analysing the expression of, cyc-like genes in species from this group. This analysis may be helped by exploiting mutants described in some species of the Compositae that affect floret development. Mutants in species such as Chrysanthemum have been described which result in a capitulum comprising only irregular flowers. These might be explained in terms of an extension of $c y c$-like gene activity into the central dome. Similarly, mutants which give only regular flowers might result from a loss of cyc-like gene activity.

These examples illustrate the importance of studying the evolution of symmetry and inflorescence architecture together. The use of comparative molecular genetic analysis of genes such as cen and $c y c$ should start to reveal how this coevolution may have occured.

\section{REFERENCES}

Alvarez, J., Guli, C. L., Yu, X. -H. \& Smyth, D. R. 1992 terminal flower: a gene affecting inflorescence development in Arabidopsis thaliana. Pl. J. 2, 103-116.

Carpenter, R. \& Coen, E. S. 1990 Floral homeotic 
mutations produced by transposon-mutagenesis in Antirrhinum majus. Genes Dev. 4, 1483-1493.

Coen, E. S., Romero, J. M., Doyle, S., Elliott, R., Murphy, G. \& Carpenter, R. 1990 floricaula: a homeotic gene required for flower development in Antirrhinum majus. Cell 63, $1311-1322$.

Linnaeus, C. 1749 De Peloria. Diss. Uppsala: Amoenitates Academy.

Olmstead, R. G, Bremer, B., Scott, K. M. \& Palmer, J. D. 1993 A parsimony analysis of the Asteridae sensu lato based on $r b c \mathrm{~L}$ sequences. Ann. Missouri Bot. Gard. 80, 700-722.

Peyritsch, J. 1870 Über Pelorienbildungen. Sitzungsber Akad. Wiss. Berlin 62, 1-27.
Peyritsch, J. 1872 Über Pelorienbildungen. Sitzungsber Akad. Wiss. Berlin 62, 1-27.

Schultz, E. A. \& Haughn, G. W. 1991 LEAFY a homeotic gene that regulates inflorescence development in Arabidopsis. Pl. Cell 3, 771-781.

Shannon, S. \& Meeks-Wagner, D. R. 1991 A mutation in the Arabidopsis TFL1 gene affects inflorescence meristem development. Pl. Cell 3, 877-892.

Stebbins, G. L. 1974 Flowering plants, evolution above the species level. Massachesetts: Harvard University Press.

Stubbe, H. 1966 Genetik und Zytologie von Antirrhinum L. sect. Antirrhinum Veb. Jena: Gustav Fischer. 